\title{
TRANSFERENCE ON CERTAIN MULTILINEAR MULTIPLIER OPERATORS
}

\author{
DASHAN FAN and SHUICHI SATO
}

(Received 20 November 1999; revised 7 June 2000)

Communicated by A. H. Dooley

\begin{abstract}
We study DeLeeuw type theorems for certain multilinear operators on the Lebesgue spaces and on the Hardy spaces. As applications, on the torus we obtain an analog of Lacey-Thiele's theorem on the bilinear Hilbert transform, as well as analogies of some recent theorems on multilinear singular integrals by Kenig-Stein and by Grafakos-Torres.
\end{abstract}

2000 Mathematics subject classification: primary 42B15, 42B20, 42B25.

\section{Introduction}

Let $\mathbb{R}^{n}$ be the $n$-dimensional Euclidean space and $\mathbb{R}^{n m}=\mathbb{R}^{n} \times \mathbb{R}^{n} \times \cdots \times \mathbb{R}^{n}$ be the $m$-fold product space. Suppose that $\mathscr{S}\left(\mathbb{R}^{n}\right)$ is the space of all Schwartz test functions on $\mathbb{R}^{n}$ and $\lambda\left(u_{1}, \ldots, u_{m}\right)$ is any function on $\mathbb{R}^{n m}$, where $u_{j} \in \mathbb{R}^{n}$ for $j=1,2, \ldots, m$. The multilinear operators $T_{\varepsilon}, \varepsilon>0$, associated with this $\lambda$ are defined by

$$
\begin{aligned}
& T_{\varepsilon}\left(f_{1}, f_{2}, \ldots, f_{m}\right)(x) \\
& \quad=\int_{\mathbb{R}^{n m}} \prod_{j=1}^{m} \hat{f_{j}}\left(u_{j}\right) \lambda\left(\varepsilon u_{1}, \ldots, \varepsilon u_{m}\right) \exp \left(2 \pi i \sum_{j=1}^{m}\left\langle u_{j}, x\right\rangle\right) d u_{1} \cdots d u_{m},
\end{aligned}
$$

for all $f_{j} \in \mathscr{S}\left(\mathbb{R}^{n}\right), j=1,2, \ldots, m$, where $\hat{f_{j}}$ is the Fourier transform of $f_{j}, x \in \mathbb{R}^{n}$ and $\left\langle u_{j}, x\right\rangle$ is the inner product of $u_{j}$ and $x$. We denote $T=T_{\varepsilon}$ if $\varepsilon=1$.

The significance of studying such kind of multilinear operators can be illustrated by following two simple model cases. First, in the case $m=1, T$ is the classical multiplier 
which plays very important roles in harmonic analysis and in partial differential equations (see [S]). Secondly, the study of the case $m>1$ is much more involved. This topic can be dated back by the pioneering work of Coifman-Meyer started from 70's [CM1, CM2, CM3, CM4], as well as some recent works by Lacey-Thiele, KenigStein and many others [KeS, CG, GK, GT, GW, LT]. Readers can see these references for more details about the background and significance in this topic. Here we list a simple example by letting $n=1, m=2$ and taking $\lambda\left(u_{1}, u_{2}\right)=\lambda\left(u_{2}-u_{1}\right)$ with $\lambda(t)=i \operatorname{sgn}(t)$, where $\operatorname{sgn}(t)$ is the sign function on $\mathbb{R}^{1}$. Then it is easy to check that

$$
T(f, g)(x)=\operatorname{pv} \pi^{-1} \int_{\mathbb{R}^{1}} f(x-t) g(x+t) t^{-1} d t
$$

is the bilinear Hilbert transform, which is related to a famous conjecture by Calderón in studying certain problems of Cauchy integrals. Very recently, Lacey and Thiele [LT, La] solved this conjecture by proving that

$$
\|T(f, g)\|_{p}<C\|f\|_{q}\|g\|_{r}
$$

provided $1 / p=1 / q+1 / r, 1<q, r \leq \infty$ and $2 / 3<p<\infty$.

Analogously, we can define multilinear operators on the torus. The $n$-torus $\mathbb{T}^{n}$ can be identified with $\mathbb{R}^{n} / \Lambda$, where $\Lambda$ is the unit lattice which is an additive group of points in $\mathbb{R}^{n}$ having integer coordinates. Let $\mathbb{T}^{n m}$ be the $m$-fold product space $\mathbb{T}^{n} \times \mathbb{T}^{n} \times \cdots \times \mathbb{J}^{n}$. The multilinear operators $\tilde{T}_{\varepsilon}, \varepsilon>0$, on $\mathbb{T}^{n m}$ associated with the function $\lambda$ are defined by

$$
\begin{aligned}
& \tilde{T}_{\varepsilon}\left(\tilde{f_{1}}, \ldots, \tilde{f_{m}}\right)(x) \\
& \quad=\sum_{k_{1} \in \Lambda} \sum_{k_{2} \in \Lambda} \cdots \sum_{k_{m} \in \Lambda} \lambda\left(\varepsilon k_{1}, \ldots, \varepsilon k_{m}\right) a_{k_{1}} a_{k_{2}} \cdots a_{k_{m}} \exp \left(2 \pi i \sum_{j=1}^{m}\left\langle k_{j}, x\right\rangle\right)
\end{aligned}
$$

for all $C^{\infty}\left(\mathbb{T}^{n}\right)$ functions

$$
\tilde{f_{j}}(x)=\sum_{k_{j} \in \Lambda} a_{k_{j}} \exp \left(2 \pi i\left\langle k_{j}, x\right\rangle\right), \quad j=1,2, \ldots, m .
$$

We denote $\tilde{T}=\tilde{T}_{\varepsilon}$ if $\varepsilon=1$.

As we mentioned before, in the case $m=1, T$ becomes the ordinary multiplier operator. One of the well-known results in that case is a theorem by DeLeeuw [L] (see also [SW, page 260]) which says that if $\lambda(u)$ is a continuous function on $\mathbb{R}^{n}$ and if $p \geq 1$, then $T$ is bounded on $L^{p}\left(\mathbb{R}^{n}\right)$ if and only if $\tilde{T}_{\varepsilon}$ is uniformly bounded on $L^{p}\left(\mathbb{T}^{n}\right)$ for $\varepsilon>0$. This theorem was later extended to many different settings. Readers can see [K, KT, AC, F, T, KaS] for further details of these generalizations.

The main purpose of this paper is to extend DeLeeuw's theorem to the case $m \geq 2$. Letting $1 / p=\sum_{j=1}^{m} 1 / p_{j}$, we will establish the following theorems. 
THEOREM 1. Suppose that $\lambda$ is an $L^{\infty}$-function which is continuous on $\mathbb{R}^{m n}$ except on a countable set. Let $T$ and $\tilde{T}_{\varepsilon}$ be the multilinear operators associated with $\lambda$. If

$$
\left\|\tilde{T}_{\varepsilon}\left(\tilde{f_{1}}, \ldots, \tilde{f_{m}}\right)\right\|_{L^{p}\left(\mathbb{T}^{n}\right)} \leq \tilde{A} \prod_{j=1}^{m}\left\|\tilde{f_{j}}\right\|_{L^{p_{j}\left(\mathbb{J}^{n}\right)}}
$$

uniformly for $\varepsilon>0$, where $\tilde{A}$ is a constant independent of $\varepsilon>0$ and $\tilde{f_{j}}$ 's, then

$$
\left\|T\left(f_{1}, \ldots, f_{m}\right)\right\|_{L^{p}\left(\mathbb{R}^{n}\right)} \leq A \prod_{j=1}^{m}\left\|f_{j}\right\|_{L^{p_{j}\left(\mathbb{R}^{n}\right)}}
$$

where $A$ is a constant independent of $f_{j}$ 's and $A \leq \tilde{A}$.

For a set $E$, denote its Lebesgue measure by $\mu(E)$. We have the following weak type theorem.

THEOREM 2. Let $T, \tilde{T}_{\varepsilon}, \lambda$ be as in Theorem 1. If

$$
\mu\left\{x \in \mathbb{T}^{n}:\left|\tilde{T}_{\varepsilon}\left(\tilde{f_{1}}, \tilde{f_{2}}, \ldots, \tilde{f_{m}}\right)(x)\right|>\alpha\right\} \leq \tilde{B}\left\{\prod_{j=1}^{m}\left\|\tilde{f_{j}}\right\|_{L^{p_{j}\left(\mathbb{T}^{n}\right)}} \alpha^{-1}\right\}^{p},
$$

where $\tilde{B}$ is independent of $\tilde{f_{j}}$ 's, $\varepsilon>0$ and $\alpha>0$, then

$$
\mu\left\{x \in \mathbb{R}^{n}:\left|T\left(f_{1}, f_{2}, \ldots, f_{m}\right)(x)\right|>\alpha\right\} \leq B\left\{\prod_{j=1}^{m}\left\|f_{j}\right\|_{L^{p_{j}}\left(\mathbb{R}^{n}\right)} \alpha^{-1}\right\}^{p},
$$

where $B \leq \tilde{B}$ is independent of $f_{j}$ 's, and $\alpha>0$.

Conversely, we have the following two theorems.

THEOREM 3. Suppose that $\lambda$ is an $L^{\infty}$-function on $\mathbb{R}^{n m}$. For a fixed $\varepsilon_{0}>0$ assume that all multi-integers $\left(k_{1}, k_{2}, \ldots, k_{m}\right) \in \Lambda \times \Lambda \times \cdots \times \Lambda$ are Lebesgue points of $\lambda\left(\varepsilon_{0} \cdot\right)$. If $(1.4)$ holds, then we have

$$
\left\|\tilde{T}_{\varepsilon_{0}}\left(\tilde{f_{1}}, \ldots, \tilde{f_{m}}\right)\right\|_{L^{p}\left(\mathbb{T}^{n}\right)} \leq \tilde{A} \prod_{j=1}^{m}\left\|\tilde{f_{j}}\right\|_{L^{p_{j}}\left(\mathbb{I}^{n}\right)}
$$

with $\tilde{A} \leq A$ being independent of $\varepsilon_{0}>0$ and $\tilde{f_{j}}$ 's.

THEOREM 4. Let $\varepsilon_{0}$ and $\lambda$ be the same as in Theorem 3. If (1.6) holds, then we have

$$
\mu\left\{x \in \mathbb{T}^{n}:\left|\tilde{T}_{\varepsilon_{0}}\left(\tilde{f_{1}}, \ldots, \tilde{f_{m}}\right)(x)\right|>\alpha\right\} \leq \tilde{B}\left\{\prod_{j=1}^{m}\left\|\tilde{f_{j}}\right\|_{L^{p_{j}\left(\mathbb{R}^{n}\right)}} \alpha^{-1}\right\}^{p},
$$

where $\tilde{B} \leq B$ is independent of $\varepsilon_{0}>0, \alpha>0$ and $\tilde{f_{j}}$ 's. 
As applications of Theorem 3, we will obtain an analog of Lacey-Thiele's theorem for bilinear conjugate Fourier series, as well as analogies of recent works on multilinear singular integrals by Kenig-Stein and Grafakos-Torres. It is worth remarking that recently Grafakos and Weiss studied an alternating definition of $T$ in a more general amenable group and obtained some other transference results similar to Theorem 3 and Theorem 4 (see [GW]). But it seems that, by their theorems, one is not able to obtain Lacey-Thiele's theorem on the torus. On the other hand, their method does not work on the $H^{p}$-spaces, which we will work on later in this paper. We also want point out a few remarks.

REMARK 1. (1) If $\lambda$ is $L^{\infty}$ and continuous on $\mathbb{R}^{n m}$, clearly $\lambda$ satisfies the condition in Theorem 3 and Theorem 4.

(2) Since the proofs for cases $m=2$ and $m>2$ are essentially the same, for the sake of simplicity, we will prove theorems for the case $m=2$. We denote $f_{1}(x)=f(x), f_{2}(x)=g(x), \tilde{f_{1}}(x)=\tilde{f}(x), \tilde{f_{2}}(x)=\tilde{g}(x)$, and $p_{1}=q, p_{2}=r$ so that $1 / p=1 / q+1 / r$ throughout this paper.

(3) The maximal operators are defined by

$$
\begin{aligned}
& T^{*}\left(f_{1}, \ldots, f_{m}\right)(x)=\sup _{\varepsilon>0}\left|T_{\varepsilon}\left(f_{1}, \ldots, f_{m}\right)(x)\right|, \\
& \tilde{T}^{*}\left(\tilde{f_{1}}, \ldots, \tilde{f_{m}}\right)(x)=\sup _{\varepsilon>0}\left|\tilde{T}_{\varepsilon}\left(\tilde{f_{1}}, \ldots, \tilde{f_{m}}\right)(x)\right| .
\end{aligned}
$$

Noting

$$
T^{*}(\cdot)(x)=\lim _{R \rightarrow \infty} \sup _{0<\varepsilon \leq R}\left|T_{\varepsilon}(\cdot)(x)\right|, \quad \tilde{T}^{*}(\cdot)(x)=\lim _{R \rightarrow \infty} \sup _{0<\varepsilon \leq R}\left|\tilde{T}_{\varepsilon}(\cdot)(x)\right|
$$

for each $x$, without any changes in the proofs of Theorem 1-Theorem 4, we may use a limit argument to obtain Theorem 1-Theorem 4 for the maximal operators.

(4) In this paper, we do not intend to pursue the study of boundedness of $T$ as those in the previous papers mentioned above. What we emphasize is to establish certain DeLeeuw type theorem, which says that, under some very mild condition, the boundedness of $T$ on the Euclidean spaces is equivalent to the boundedness of its corresponding family $\left\{\tilde{T}_{\varepsilon}\right\}$ on the torus, so that one can easily obtain an analogous theorem on $\tilde{T}$ as soon as a new theorem of $T$ is obtained.

(5) In the direction of generalization, one might expect to formulate a theorem that transfers not only the bilinear Hilbert transform, but also the multilinear fractional integrals in [KeS] and [GT]. It is known that, in general, DeLeeuw's theorem fails even in the one parameter case if $p \neq q$ (for example see [KaS]). So one might need some extra condition to establish such a theorem.

(6) Following the ideas in $[\mathrm{AC}]$ and [T], it is possible to establish transference theorem of multilinear operators between $\mathbb{R}^{n}$ and $\mathbb{Z}^{n}$. The proof for this case is in a different style, to avoid that this paper becomes too long, we will study this problem in our future papers. 
The proofs of Theorem 1 and Theorem 2 use a standard argument involving the definition of Riemann integrals (see [SW]). For completeness, we present them in the second section. However, the duality argument used to prove Theorem 3 for the case $m=1$ (see [SW, page 260]) is difficult to adopt. We use an alternating method to study Theorem 3 and Theorem 4 in Section 3. In Section 4, we study DeLeeuw's theorem on the Hardy spaces by using the atomic characterization of $H^{p}$.

Finally, in this paper, we use letter ' $\mathrm{C}$ ' to denote (possibly different) constants that are independent of the essential variables in the argument.

\section{Proofs of Theorem 1 and Theorem 2}

Let $\mathscr{D}\left(\mathbb{R}^{n}\right)=\left\{f \in \mathscr{S}\left(\mathbb{R}^{n}\right): f\right.$ has compact support $\}$. The space $\mathscr{D}\left(\mathbb{R}^{n}\right)$ is dense in the space $L^{p}\left(\mathbb{R}^{n}\right)$, so it is enough to show the theorem for functions $f, g \in \mathscr{D}\left(\mathbb{R}^{n}\right)$. In order to do so, define $\tilde{f_{\varepsilon}}$ and $\tilde{g}_{\varepsilon}$, for $\varepsilon>0$, to be the dilated and periodized versions of $f$ and $g$, viz

$$
\tilde{f_{\varepsilon}}=\varepsilon^{-n} \sum_{k \in \Lambda} f\left(\frac{x+k}{\varepsilon}\right), \quad \tilde{g}_{\varepsilon}=\varepsilon^{-n} \sum_{l \in \Lambda} g\left(\frac{x+l}{\varepsilon}\right)
$$

Then by the Poisson summation formula we obtain

$$
\tilde{f_{\varepsilon}}=\sum_{k \in \Lambda} \hat{f}(\varepsilon k) e^{2 \pi i(k, x)}, \quad \tilde{g}_{\varepsilon}=\sum_{l \in \Lambda} \hat{g}(\varepsilon l) e^{2 \pi i(l, x)} .
$$

By the definition of the Riemann integral (see also [SW]), we know that

$$
\lim _{\varepsilon \rightarrow 0} \varepsilon^{2 n} \tilde{T}_{\varepsilon}\left(\tilde{f_{\varepsilon}}, \tilde{g}_{\varepsilon}\right)(\varepsilon x)=T(f, g)(x) .
$$

Let

$$
Q=\left\{x \in \mathbb{R}^{n}:-1 / 2 \leq x_{j}<1 / 2, j=1,2 \ldots, n\right\}
$$

be the fundamental cube on which

$$
\int_{\mathbb{T}_{n}} \tilde{f}(x) d x=\int_{Q} \tilde{f}(x) d x
$$

for all function $\tilde{f}$ on $\mathbb{T}^{n}$. We choose $\{\varepsilon\}$ as a discrete sequence going to 0 .

In order to prove Theorem 1 , we choose $\eta(x) \geq 0$ to be a function in $\mathscr{D}\left(\mathbb{R}^{n}\right)$ satisfying $\eta(0)=1$ and $\sum_{m \in \Lambda} \eta(x+m)=1$. By Fatou's lemma, we have

$$
\|T(f, g)\|_{L^{p}\left(\mathbb{R}^{n}\right)}^{p} \leq \liminf _{\varepsilon \rightarrow 0} \int_{\mathbb{R}^{n}} \eta(\varepsilon x)\left|\varepsilon^{2 n} \tilde{T}_{\varepsilon}\left(\tilde{f}_{\varepsilon}, \tilde{g}_{\varepsilon}\right)(\varepsilon x)\right|^{p} d x .
$$


By changing variables on $x$ and using the fact $\sum \eta(x+k)=1$, it is easy to see that

$$
\begin{aligned}
\|T(f, g)\|_{L^{p}\left(\mathbb{R}^{n}\right)} & \leq \liminf _{\varepsilon \rightarrow 0}\left\{\varepsilon^{2 n p-n} \int_{\mathbb{R}^{n}} \eta(x)\left|\tilde{T}_{\varepsilon}\left(\tilde{f_{\varepsilon}}, \tilde{g}_{\varepsilon}\right)(x)\right|^{p} d x\right\}^{1 / p} \\
& =\liminf _{\varepsilon \rightarrow 0} \varepsilon^{2 n-n / p}\left\|\tilde{T}_{\varepsilon}\left(\tilde{f_{\varepsilon}}, \tilde{g}_{\varepsilon}\right)\right\|_{L^{p}\left(\mathbb{J}^{n}\right)^{.}}
\end{aligned}
$$

By the assumption, we have that

$$
\|T(f, g)\|_{L^{p}\left(\mathbb{R}^{n}\right)} \leq \tilde{A} \liminf _{\varepsilon \rightarrow 0} \varepsilon^{2 n-n / p}\left\|\tilde{f_{\varepsilon}}\right\|_{L^{q}\left(\mathbb{J}^{n}\right)}\left\|\tilde{g}_{\varepsilon}\right\|_{L^{r}\left(\mathbb{T}^{n}\right)} .
$$

Finally, by [SW, page 266], we know that if $\varepsilon$ is sufficiently small, then

$$
\left\|\tilde{f_{\varepsilon}}\right\|_{L^{q}\left(\mathbf{I}^{n}\right)}=\varepsilon^{-n+n / q}\|f\|_{L^{q}\left(\mathbb{J}^{n}\right)}, \quad\left\|\tilde{g}_{\varepsilon}\right\|_{L^{\prime}\left(\mathbb{J}^{n}\right)}=\varepsilon^{-n+n / r}\|g\|_{L^{\prime}\left(\mathbb{T}^{n}\right)} .
$$

Thus we obtain

$$
\|T(f, g)\|_{L^{p}\left(\mathbb{R}^{n}\right)} \leq \tilde{A}\|f\|_{L^{q}\left(\mathbb{T}^{n}\right)}\|g\|_{L^{r}\left(\mathbb{T}^{n}\right)} .
$$

Theorem 1 is proved.

We now turn to prove Theorem 2. Let $\chi_{Q}(x)$ be the characteristic function of $Q$. By Fatou's lemma, we have

$$
\begin{aligned}
\mu\{x & \left.\in \mathbb{R}^{n}:|T f(x)|>\alpha\right\} \\
& \leq \liminf _{\varepsilon \rightarrow 0} \mu\left\{x \in \mathbb{R}^{n}, \chi_{Q}(\varepsilon x)\left|\varepsilon^{2 n} \tilde{T}_{\varepsilon}\left(\tilde{f_{\varepsilon}}, \tilde{g}_{\varepsilon}\right)(\varepsilon x)\right|>\alpha\right\} \\
& =\liminf _{\varepsilon \rightarrow 0} \varepsilon^{-n} \mu\left\{x \in \mathbb{R}^{n}, \chi_{Q}(x)\left|\varepsilon^{2 n} \tilde{T}_{\varepsilon}\left(\tilde{f_{\varepsilon}}, \tilde{g}_{\varepsilon}\right)(x)\right|>\alpha\right\} \\
& =\liminf _{\varepsilon \rightarrow 0} \varepsilon^{-n} \mu\left\{x \in Q,\left|\tilde{T}_{\varepsilon}\left(\tilde{f_{\varepsilon}}, \tilde{g}_{\varepsilon}\right)(x)\right|>\alpha \varepsilon^{-2 n}\right\} \\
& \leq \liminf _{\varepsilon \rightarrow 0} \tilde{B} \varepsilon^{-n}\left\{\left\|\tilde{f_{\varepsilon}}\right\|_{L^{q}\left(\mathbb{T}^{n}\right)}\left\|\tilde{g}_{\varepsilon}\right\|_{L^{\prime}\left(\mathbb{J}^{n}\right)} \alpha^{-1} \varepsilon^{2 n}\right\}^{p} \\
& =\tilde{B}\left\{\|f\|_{L^{q}\left(\mathbb{J}^{n}\right)}\|g\|_{L^{\prime}\left(\mathbb{T}^{n}\right)} \alpha^{-1}\right\}^{p},
\end{aligned}
$$

which proves Theorem 2 .

\section{Proofs of Theorem 3 and Theorem 4}

Let $\left\|T_{\varepsilon}\right\|=\sup \left\{\left\|T_{\varepsilon}(f, g)\right\|_{p}:\|f\|_{q}=\|g\|_{r}=1\right\}$. It is easy to see that $\left\|T_{\varepsilon}\right\|=\|T\|$ for all $\varepsilon>0$. So to prove Theorem 3, without loss of generality, we may assume $\varepsilon_{0}=1$ (we may make the same assumption in proving Theorem 4 , for the same reason). 
Fix a positive integer $K$, define the set $\Omega_{K}$ by

$$
\Omega_{K}=[-1 / 2-1 / K, 1 / 2+1 / K]^{n} .
$$

Let $\Psi$ be a function in $\mathscr{S}\left(\mathbb{R}^{n}\right)$ satisfying supp $\Psi \subseteq \Omega_{K}, 0 \leq \Psi(x) \leq 1$, and $\Psi(x) \equiv 1$ on $Q$. We denote $\Psi^{1 / N}(x)=\Psi(x / N)$ for an integer $N$. For any $C^{\infty}$ functions $\tilde{f}(x)=\sum_{k \in \Lambda} a_{k} e^{2 \pi i(k, x)}$ and $\tilde{g}(x)=\sum_{\nu \in \Lambda} b_{\nu} e^{2 \pi i\langle\nu, x)}$, we let

$$
E_{N}(\tilde{f}, \tilde{g})(x)=\Psi(x / N)^{2} \tilde{T}(\tilde{f}, \tilde{g})(x)-T\left(\Psi^{1 / N} \tilde{f}, \Psi^{1 / N} \tilde{g}\right)(x) .
$$

By checking the Fourier transform, it is easy to see that

$$
\begin{aligned}
-E_{N}(\tilde{f}, \tilde{g})(x)= & \sum_{k \in \Lambda} \sum_{v \in \Lambda} a_{k} b_{\nu} e^{2 \pi i(k+v, x\rangle} \int_{\mathbb{R}^{n}} \int_{\mathbb{R}^{n}} \hat{\Psi}(u) \hat{\Psi}(v)[\lambda(k+u / N, \nu+v / N) \\
& -\lambda(k, v)\} e^{2 \pi i(u, x / N)} e^{2 \pi i\langle v, x / N\rangle} d u d v
\end{aligned}
$$

Since $\left\{a_{k}\right\},\left\{b_{v}\right\}$ decay rapidly, $\lambda$ is $L^{\infty}$ and all $(k, v)$ are Lebesgue points of $\lambda$, clearly $E_{N}(\tilde{f}, \tilde{g})(x) \rightarrow 0$ uniformly for $x \in \mathbb{R}^{n}$ as $N \rightarrow \infty$.

Noting $\tilde{T}(\tilde{f}, \tilde{g})(x)$ is a periodic function, we have

$$
\|\tilde{T}(\tilde{f}, \tilde{g})\|_{L^{p}\left(\mathbb{J}^{n}\right)}=\left\{N^{-n} \int_{N Q}|\tilde{T}(\tilde{f}, \tilde{g})|^{p} d x\right\}^{1 / p} .
$$

By the choice of $\Psi$, we further obtain

$$
\|\tilde{T}(\tilde{f}, \tilde{g})\|_{L^{p}\left(\mathbf{T}^{n}\right)}=\left\{N^{-n} \int_{N Q}\left|\Psi(x / N)^{2} \tilde{T}(\tilde{f}, \tilde{g})\right|^{p} d x\right\}^{1 / p} .
$$

Thus by (3.1), we have that if $p \geq 1$, then

$$
\begin{aligned}
\|\tilde{T}(\tilde{f}, \tilde{g})\|_{L^{p}\left(\mathbf{J}^{n}\right)} \leq & \left\{N^{-n} \int_{\mathbb{R}^{n}}\left|T\left(\Psi^{1 / N} \tilde{f}, \Psi^{1 / N} \tilde{g}\right)\right|^{p} d x\right\}^{1 / p} \\
& +\left\{N^{-n} \int_{N Q}\left|E_{N}(\tilde{f}, \tilde{g})(x)\right|^{p} d x\right\}^{1 / p},
\end{aligned}
$$

and that the second integral on the right-hand side of the above inequality goes to zero as $N \rightarrow \infty$. On the other hand, the first integral on the right-hand side of the above inequality is equal to

$$
N^{-n / p}\left\|T\left(\Psi^{1 / N} \tilde{f}, \Psi^{1 / N} \tilde{g}\right)\right\|_{L^{p}\left(\mathbb{R}^{n}\right)}
$$


Thus by the assumption and the choice of $\Psi$, it is bounded by

$$
\begin{aligned}
& N^{-n / p} A\left\|\Psi^{1 / N} \tilde{f}\right\|_{L^{q}\left(\mathbf{R}^{n}\right)}\left\|\Psi^{1 / N} \tilde{\boldsymbol{g}}\right\|_{L^{r}\left(\mathbb{R}^{n}\right)} \\
& \quad \leq A N^{-n / p}\left\{\int_{N \Omega_{K}}|\tilde{f}(x)|^{q} d x\right\}^{1 / q}\left\{\int_{N \Omega_{K}}|\tilde{g}(x)|^{r} d x\right\}^{1 / r},
\end{aligned}
$$

where $N \Omega_{K}=[-N / 2-N / K, N / 2+N / K]^{n}$. Choose $N$ such that $N / K$ are integers. Then as $N \rightarrow \infty$ we have, since $\tilde{f}$ and $\tilde{g}$ are periodic functions, that

$$
\begin{aligned}
\|\tilde{T}(\tilde{f}, \tilde{g})\|_{L^{p}\left(\mathbf{T}^{n}\right)} \leq & A N^{-n / p}\left\{(N+2 N / K)^{n} \int_{Q}|\tilde{f}(x)|^{q} d x\right\}^{1 / q} \\
& \times\left\{(N+2 N / K)^{n} \int_{Q}|\tilde{g}(x)|^{r} d x\right\}^{1 / r}+o(1) \\
= & A(1+2 / K)^{n /(1 / q+1 / r)}\|\tilde{f}\|_{L^{p}\left(\mathbb{T}^{n}\right)}\|\tilde{g}\|_{L^{p}\left(\mathbf{T}^{n}\right)}+o(1) .
\end{aligned}
$$

Letting first $N \rightarrow \infty$, then $K \rightarrow \infty$, we prove Theorem 3 for $p \geq 1$.

For $0<p<1$, we have

$$
\begin{aligned}
\|\tilde{T}(\tilde{f}, \tilde{g})\|_{L^{p}\left(T^{n}\right)}^{p} \leq & N^{-n} \int_{\mathbb{R}^{n}}\left|T\left(\Psi^{1 / N} \tilde{f}, \Psi^{1 / N} \tilde{g}\right)(x)\right|^{p} d x \\
& +N^{-n} \int_{N Q}\left|E_{N}(\tilde{f}, \tilde{g})(x)\right|^{p} d x .
\end{aligned}
$$

Thus the proof is the same as that for $p \geq 1$.

To prove Theorem 4 , fixing any $\alpha>0$, we have

$$
\begin{aligned}
\mu\{x \in Q:|\tilde{T}(\tilde{f}, \tilde{g})(x)|>\alpha\} & =N^{-n} \mu\{x \in N Q:|\tilde{T}(\tilde{f}, \tilde{g})(x)|>\alpha\} \\
& =N^{-n} \mu\left\{x \in N Q:\left|\Psi(x / N)^{2} \tilde{T}(\tilde{f}, \tilde{g})(x)\right|>\alpha\right\}
\end{aligned}
$$

Thus by (3.1) and the fact that $E_{N}(\tilde{f}, \tilde{g})(x) \rightarrow 0$ uniformly in $x$ as $N \rightarrow \infty$, for any $\beta \in(0, \alpha)$ we can choose $N$ sufficiently large such that

$$
\mu\{x \in Q:|\tilde{T}(\tilde{f}, \tilde{g})(x)|>\alpha\} \leq N^{-n} \mu\left\{x \in \mathbb{R}^{n}:\left|T\left(\Psi^{1 / N} \tilde{f}, \Psi^{1 / N} \tilde{g}\right)(x)\right|>\alpha-\beta\right\} .
$$

Thus by the assumption we have

$$
\begin{aligned}
\mu\{x & \in Q:|\tilde{T}(\tilde{f}, \tilde{g})(x)|>\alpha\} \\
& \leq N^{-n} B\left\{\left\|\Psi^{1 / N} \tilde{f}\right\|_{L^{q}\left(\mathbb{R}^{n}\right)}\left\|\Psi^{1 / N} \tilde{g}\right\|_{L^{\prime}\left(\mathbb{R}^{n}\right)}(\alpha-\beta)^{-1}\right\}^{p} \\
& \leq B\left\{\|\tilde{f}\|_{L^{q}\left(\mathbf{I}^{n}\right)}\|\tilde{g}\|_{L^{\prime}\left(\mathbf{I}^{n}\right)}(\alpha-\beta)^{-1}\right\}^{p}(1+2 / K)^{n(1 / q+1 / r) p} .
\end{aligned}
$$


Since $\beta>0$ is arbitrary, letting $K \rightarrow \infty$, the theorem is proved.

We now present some applications of Theorem 3. First we consider the bilinear Hilbert transform on the one-dimensional torus

$$
\tilde{H}(\tilde{f}, \tilde{g})(x)=\text { p.v. } \int_{-1 / 2}^{1 / 2} \tilde{f}(x-t) \cot (\pi t) \tilde{g}(t+x) d t .
$$

Then it is easy to check

$$
\tilde{H}(\tilde{f}, \tilde{g})(x)=\sum_{-\infty}^{\infty} \sum_{-\infty}^{\infty} a_{k} b_{\nu} i \operatorname{sgn}(v-k) e^{2 \pi i(k+v) x} .
$$

By the known result of $H(f, g)$ (see [LT, La]) and the proof of Theorem 3 we have

COROLLARY 1. $\tilde{H}$ maps $L^{q}\left(\mathbb{T}^{1}\right) \times L^{r}\left(\mathbb{T}^{1}\right)$ into $L^{p}\left(\mathbb{T}^{1}\right)$ for $1<q, r \leq \infty, 2 / 3<$ $p<\infty$ and $1 / p=1 / q+1 / r$.

Proof. Let $\lambda(u, v)=i \operatorname{sgn}(v-u)$ and $E_{N}(\tilde{f,} \tilde{g})$ be as in the proof of Theorem 3 . Note that $\lambda(u, v)=-\lambda(v, u)$. Therefore, by symmetry we have

$$
\int_{\mathbb{R}} \int_{\mathbb{R}} \hat{\Psi}(u) \hat{\Psi}(v) \lambda(k+u / N, k+v / N) e^{2 \pi i(u, x / N)} e^{2 \pi i\langle v, x / N)} d u d v=0
$$

for all $k \in \Lambda, x \in \mathbb{R}$ and $N$. So, though the points $(k, k) \in \Lambda$ are not the Lebesgue points of $\lambda(u, v)$, we have $E_{N}(\tilde{f}, \tilde{g})(x) \rightarrow 0$ uniformly in $x \in \mathbb{R}$ as $N \rightarrow \infty$. Thus, by the proof of Theorem 3 and [LT, La], we get the corollary.

Secondly, we recall the multilinear singular integrals $T_{K}$ on $\mathbb{R}^{n m}$ :

$$
T_{K}\left(f_{1}, \ldots, f_{m}\right)(x)=\text { p.v. } \int_{\mathbb{R}^{n m}} f_{1}\left(x-y_{1}\right) \cdots f_{m}\left(x-y_{m}\right) K\left(y_{1}, \ldots, y_{m}\right) d y_{1} \cdots d y_{m},
$$

where $K$ is the Calderón-Zygmund kernel (see [GT, KeS]). We define

(3.2) $\tilde{T}_{K}\left(\tilde{f_{1}}, \ldots, \tilde{f_{m}}\right)(x)=\sum_{k_{1}, \ldots, k_{m}} \hat{K}\left(k_{1}, \ldots, k_{m}\right) a_{k_{1}} a_{k_{2}} \cdots a_{k_{m}} \exp \left(2 \pi i \sum_{j=1}^{m}\left\langle k_{j}, x\right\rangle\right)$,

where $\left(k_{1}, \ldots, k_{m}\right)$ ranges over $\Lambda^{m} \backslash\{(0, \ldots, 0)\}$.

COROLLARY 2. Let $K$ be a locally integrable function on $\mathbb{R}^{n m}-\{0\}$ which satisfies the size condition

$$
\left|K\left(u_{1}, \ldots, u_{m}\right)\right| \leq C\left|\left(u_{1}, \ldots, u_{m}\right)\right|^{-n m},
$$


the cancellation condition

$$
\left|\int_{R_{1}<\left|\left(u_{1}, \ldots, u_{m}\right)\right|<R_{2}} K\left(u_{1}, \ldots, u_{m}\right) d u_{1} \cdots d u_{m}\right| \leq C<\infty,
$$

for all $0<R_{1}<R_{2}<\infty$, and the smoothness condition

$$
\begin{aligned}
& \left|K\left(u_{1}, \ldots, u_{j}, \ldots, u_{m}\right)-K\left(u_{1}, \ldots, u_{j}^{\prime}, \ldots, u_{m}\right)\right| \\
& \quad \leq C \frac{\left|u_{j}-u_{j}^{\prime}\right|^{\delta}}{\left|\left(u_{1}, \ldots, u_{m}\right)\right|^{n m+\delta}}
\end{aligned}
$$

for some $\delta>0$ whenever $\left|u_{j}-u_{j}^{\prime}\right|<\left|u_{j}\right| / 2$. Suppose that for some monotonically decreasing sequence $\varepsilon_{j}$ convergent to zero, the limit

$$
\lim _{j \rightarrow \infty} \int_{\varepsilon_{j}<\left|\left(u_{1}, \ldots, u_{m}\right)\right| \leq 1} K\left(u_{1}, \ldots, u_{m}\right) d u_{1} \cdots d u_{m}
$$

exists. Then $\tilde{T}_{K}$ maps $L^{p_{1}}\left(\mathbb{T}^{n}\right) \times \cdots \times L^{p_{m}}\left(\mathbb{T}^{n}\right)$ into $L^{p}\left(\mathbb{T}^{n}\right)$ with $1<p_{j}<\infty$, $1 / m<p<\infty$ and $1 / p=\sum_{j=1}^{m} 1 / p_{j}$.

PROOF. We prove the corollary for the case $m=2$. The proofs for the other cases are essentially the same as that for the case $m=2$. From [GT], we know that $\|\hat{K}\|_{\infty} \leq C<\infty$. Thus by (3.4)-(3.6) it is easy to see that $\hat{K}$ is a continuous function on $\mathbb{R}^{2 n} \backslash\{0\}$. We write

$$
\begin{aligned}
& \tilde{f}(x)=a_{0}+\sum_{k \neq 0} a_{k} e^{2 \pi i(k, x)}=a_{0}+\tilde{f_{1}}(x), \\
& \tilde{g}(x)=b_{0}+\sum_{k \neq 0} b_{k} e^{2 \pi i\langle k, x\rangle}=b_{0}+\tilde{g}_{1}(x),
\end{aligned}
$$

Then,

$$
\tilde{T}_{K}(\tilde{f}, \tilde{g})(x)=\tilde{T}_{K}\left(a_{0}, \tilde{g}_{1}\right)(x)+\tilde{T}_{K}\left(\tilde{f_{1}}, b_{0}\right)(x)+\tilde{T}_{K}\left(\tilde{f_{1}}, \tilde{g}_{1}\right)(x) .
$$

Let $E_{N}(\tilde{f}, \tilde{g})(x)$ be defined as in (3.1) with $T_{K}$ and $\tilde{T}_{K}$ in place of $T$ and $\tilde{T}$, respectively. Since $\hat{K}$ is continuous on $\mathbb{R}^{2 n} \backslash\{0\}$, we see that $E_{N}\left(a_{0}, \tilde{g}_{1}\right)(x) \rightarrow 0$, $E_{N}\left(\tilde{f_{1}}, b_{0}\right)(x) \rightarrow 0, E_{N}\left(\tilde{f_{1}}, \tilde{g}_{1}\right)(x) \rightarrow 0$ uniformly in $x \in \mathbb{R}^{n}$ as $N \rightarrow \infty$. Thus by the proof of Theorem 3 and Theorem 5 in [GT], we have

$$
\begin{aligned}
\left\|\tilde{T}_{K}(\tilde{f}, \tilde{g})\right\|_{L^{p}\left(\mathbb{J}^{n}\right)} \leq & C\left\|a_{0}\right\|_{L^{p_{1}}\left(\mathbb{J}^{n}\right)}\left\|\tilde{g}_{1}\right\|_{L^{p_{2}\left(\mathbb{T}^{n}\right)}}+C\left\|\tilde{f_{1}}\right\|_{L^{p_{1}\left(\mathbb{T}^{n}\right)}}\left\|b_{0}\right\|_{L^{p_{2}\left(\mathbb{J}^{n}\right)}} \\
& +C\left\|\tilde{f_{1}}\right\|_{L^{p_{1}}\left(\mathbb{T}^{n}\right)}\left\|\tilde{g}_{1}\right\|_{L^{p_{2}\left(\mathbb{J}^{n}\right)}} .
\end{aligned}
$$

This proves the corollary, since

$$
\begin{array}{ll}
\left\|a_{0}\right\|_{L^{p_{1}\left(\mathbb{J}^{n}\right)}} \leq\|\tilde{f}\|_{L^{p_{1}\left(\mathbb{J}^{n}\right)}}, & \left\|\tilde{f_{1}}\right\|_{L^{p_{1}\left(\mathbb{J}^{n}\right)}} \leq 2\|\tilde{f}\|_{L^{p_{1}\left(\mathbb{I}^{n}\right)}}, \\
\left\|b_{0}\right\|_{L^{p_{2}\left(\mathbb{J}^{n}\right)}} \leq\|\tilde{g}\|_{L^{p_{2}\left(\mathbb{J}^{n}\right)}}, \quad\left\|\tilde{g_{1}}\right\|_{L^{p_{2}\left(\mathbb{I}^{n}\right)}} \leq 2\|\tilde{g}\|_{L^{p_{2}\left(\mathbb{J}^{n}\right)}} .
\end{array}
$$


REMARK 2. Theorem 5 in [GT] was studied by Coifman-Meyer [CM1] if $\mathrm{K}$ is the kernel in the following Corollary 3.

COROLlARY 3. Suppose that $K$ is homogeneous of degree $-n m$, smooth away from the origin, and has mean value 0 on the unit sphere in $\mathbb{R}^{n m}$. Then $\tilde{T}_{K}$ maps $L^{p_{1}}\left(\mathbb{T}^{n}\right) \times \cdots \times L^{p_{m}}\left(\mathbb{J}^{n}\right)$ into $L^{p}\left(\mathbb{T}^{n}\right)$, with $1 / m<p<\infty, 1<p_{j}<\infty$ and $1 / p=\sum_{j=1}^{m} 1 / p_{j}$.

PROOF. Clearly, $K$ satisfies (3.3)-(3.6) so that by Corollary 2, we obtain Corollary 3 . Here we note that for the kernel considered in this corollary, the boundedness of $T_{K}$ used in the proof of Corollary 2 also comes from [KeS, Theorem 8].

REMARK 3. It is possible to extend Theorem 1 and Theorem 3 to the Lorentz spaces so that we can obtain some weak-type estimates for $\tilde{T}_{K}$, which are analogous to those in [GT, Theorem 5] and [KeS, Theorem 8].

\section{Bilinear operators in Hardy spaces}

For $j=1,2, \ldots, \gamma$, let $\lambda_{j}$ and $\mu_{j}$ be bounded functions on $\mathbb{R}^{n}$. Let $U_{j}$ and $\tilde{U}_{j}$ be multipliers associate to $\lambda_{j}$ on $\mathbb{R}^{n}$ and $\mathbb{T}^{n}$, respectively; $V_{j}$ and $\tilde{V}_{j}$ be multipliers associate to $\mu_{j}$ on $\mathbb{R}^{n}$ and $\mathbb{T}^{n}$, respectively.

The bilinear operators $B_{\gamma}(f, g)(x)$ is defined by, for any $f, g \in \mathscr{S}\left(\mathbb{R}^{n}\right)$,

$$
B_{\gamma}(f, g)(x)=\sum_{j=1}^{\gamma} U_{j}(f)(x) V_{j}(g)(x) .
$$

Similarly, the operator $\tilde{B}_{\gamma}$ is defined by

$$
\tilde{B}_{\gamma}(\tilde{f}, \tilde{g})(x)=\sum_{j=1}^{\gamma} \tilde{U}_{j}(\tilde{f})(x) \tilde{V}_{j}(\tilde{g})(x)
$$

for all

$$
\tilde{f}(x)=\sum_{k \in \Lambda} a_{k} e^{2 \pi i(k, x)} \in C^{\infty}\left(\mathbb{T}^{n}\right), \quad \tilde{g}(x)=\sum_{\nu \in \Lambda} b_{\nu} e^{2 \pi i(v, x)} \in C^{\infty}\left(\mathbb{T}^{n}\right),
$$

where

$$
\tilde{U}_{j}(\tilde{f})(x)=\sum_{k \in \Lambda} a_{k} \lambda_{j}(k) e^{2 \pi i(k, x)}, \quad \tilde{V}_{j}(\tilde{g})(x)=\sum_{\nu \in \Lambda} b_{\nu} \mu_{j}(\nu) e^{2 \pi i\langle\nu, x\rangle} .
$$

The boundedness of bilinear operator $B_{\gamma}(f, g)$ on the Hardy spaces was studied by Coifman and Grafakos in [CG] (actually, in their study, $U_{j}$ 's and $V_{j}$ 's can be general Calderón-Zygmund operators of non-convolution type). Since there is no essential difference between $\gamma=1$ and $\gamma>1$, for simplicity, we study the case $\gamma=1$. By the 
definition, it is easy to see that if $\gamma=1$, then $B_{1}(f, g)$ is a special case of $T(f, g)$ and $\tilde{B}_{1}(\tilde{f}, \tilde{g})$ is a special case of $\tilde{T}(\tilde{f}, \tilde{g})$ with $\lambda(u, v)=\lambda_{1}(u) \mu_{1}(v)$. Therefore, naturally we will study DeLeeuw's theorem for $T(f, g)$ on the Hardy spaces. Below we first review the definition of the Hardy spaces.

Let $H^{p}\left(\mathbb{R}^{n}\right), 0<p<\infty$, be the Hardy spaces defined by [FS]

$$
H^{p}\left(\mathbb{R}^{n}\right)=\left\{f \in \mathscr{S}^{\prime}\left(\mathbb{R}^{n}\right),\left\|\Phi^{+} f\right\|_{L^{p}\left(\mathbb{R}^{n}\right)}<\infty\right\},
$$

where $\Phi^{+} f(x)=\sup _{t>0}\left|\Phi_{t} * f(x)\right|, \Phi_{t}(x)=t^{-n} \Phi(x / t)$, and $\Phi \in \mathscr{S}\left(\mathbb{R}^{n}\right)$ is a radial function satisfying $\int \Phi=1$. The corresponding periodic Hardy spaces are $H^{p}\left(\mathbb{T}^{n}\right)=\left\{\tilde{f} \in \mathscr{S}^{\prime}\left(\mathbb{J}^{n}\right),\left\|\tilde{\Phi}^{+} \tilde{f}\right\|_{L^{p}\left(\mathbb{R}^{n}\right)}<\infty\right\}$, where $\tilde{\Phi}^{+} \tilde{f}(x)=\sup _{t>0}|\tilde{\Phi}, * \tilde{f}(x)|$, $\tilde{\Phi}_{t}(x)=\sum_{k \in \Lambda} \hat{\Phi}(t k) e^{2 \pi i(k, x)}+C t^{-n} \sum_{k \in \Lambda} \Phi((x+k) / t)$.

In this section we will establish the following theorem.

THEOREM 5. Let $\lambda$ be a continuous and bounded functions on $\mathbb{R}^{n}$, and $T(f, g)$ and $\tilde{T}(\tilde{f}, \tilde{g})$ be the same as in Section 3. Suppose $1 / p=1 / q+1 / r$. If there is a $C>0$ such that $\|T(f, g)\|_{H^{p}\left(\mathbb{R}^{n}\right)} \leq C\|f\|_{H^{q}\left(\mathbb{R}^{n}\right)}\|g\|_{H^{r}\left(\mathbb{R}^{n}\right)}$ for all $f \in H^{q}\left(\mathbb{R}^{n}\right) \cap \mathscr{S}\left(\mathbb{R}^{n}\right)$ and $g \in H^{r}\left(\mathbb{R}^{n}\right) \cap \mathscr{S}\left(\mathbb{R}^{n}\right)$, then $\|\tilde{T}(\tilde{f}, \tilde{g})\|_{H^{p}\left(\mathbb{T}^{n}\right)} \leq C\|\tilde{f}\|_{H^{q}\left(\mathbb{T}^{n}\right)}\|\tilde{g}\|_{H^{r}\left(\mathbb{J}^{n}\right)}$ for all $f, g \in C^{\infty}\left(\mathbb{T}^{n}\right)$.

To prove Theorem 5, we need to use the atomic characterization of the Hardy space. A regular $(p, 2, s)$ atom is a function $\alpha(x)$ supported in some ball $B\left(x_{0}, \rho\right)$ satisfying:

(i) $\|\alpha\|_{2} \leq \rho^{-n / p+n / 2}$;

(ii) $\int_{\mathbb{R}^{n}} \alpha(x) P(x) d x=0$

for all polynomials $P(x)$ of degree less than or equal to $s$.

The space $H_{a}^{p, s}\left(\mathbb{R}^{n}\right), 0<p \leq 1$, is the space of all distributions $f \in \mathscr{S}^{\prime}\left(\mathbb{R}^{n}\right)$ having the form

$$
f=\sum c_{k} \alpha_{k}
$$

and satisfying

$$
\sum\left|c_{k}\right|^{p}<\infty
$$

where each $\alpha_{k}$ is a $(p, 2, s)$ atom. The 'norm' $\|f\|_{H_{a}^{p . s}\left(\mathbb{R}^{n}\right)}$ is the infimum of all expressions $\left(\sum\left|c_{k}\right|^{p}\right)^{1 / p}$ for which we have a representation (4.3) of $f$. A wellknown fact (see [FS]) is that $\|f\|_{H_{a}^{p . s}\left(\mathbb{R}^{n}\right)} \cong\|f\|_{H^{p}\left(\mathbb{R}^{n}\right)}$ and in particular, $\|a\|_{H^{p}\left(\mathbb{R}^{n}\right)} \leq C$, with a constant $C$ independent of the $(p, 2, s)$ atom $a(x)$ if $s \geq[n(1 / p-1)]$.

We also have a similar decomposition theorem for any function $\tilde{f} \in H^{p}\left(\mathbb{T}^{n}\right)$. In particular, suppose $\tilde{f} \in C^{\infty}\left(\mathbb{T}^{n}\right)$ and its Fourier coefficient

$$
a_{0}(\tilde{f})=\int_{Q} \tilde{f}(x) d x=0 .
$$


Then we have the following lemma.

LEMMA 1. Suppose $\tilde{f} \in C^{\infty}\left(\mathbb{T}^{n}\right)$ with $a_{0}(\tilde{f})=0$. If we restrict $x$ to $Q$, then for any fixed positive integer $s$

$$
\tilde{f}(x)=\sum c_{k} \alpha_{k}(x)
$$

where each $\alpha_{k}(x)$ is a $(p, 2, s)$ atom satisfying $\alpha_{k}(x+l)=\alpha_{k}(x)$ for $l \in \Lambda$, and $\|\tilde{f}\|_{H^{p}\left(\mathbb{(}^{n}\right)}^{p} \cong \sum\left|c_{k}\right|^{p}$.

The proof can be found in $[\mathrm{BF}]$.

Now we are in a position to prove Theorem 5. For any $\tilde{f}, \tilde{g} \in C^{\infty}\left(\mathbb{T}^{n}\right)$, we have

$$
\tilde{f}(x)=\sum_{k \in \Lambda} a_{k} e^{2 \pi i(k, x)} \quad \text { and } \quad \tilde{g}(x)=\sum_{\nu \in \Lambda} b_{\nu} e^{2 \pi i(\nu, x)}
$$

with rapidly decaying coefficients. Recalling that $0<p \leq q$ and $0<p \leq r$ and a well-known fact $H^{p}=L^{p}$ if $p>1$, we can use the same argument as in proving Theorem 3 to prove Theorem 5 in the case $p>1$. It now suffices to show the case $0<p \leq 1$ and $0<q, r \leq 1$, the case $0<p \leq q \leq 1<r$ and the case $0<p \leq 1<q \leq r$. We prove these three cases separately.

CASE $1.0<p \leq 1<q \leq r$. In this case $H^{q}=L^{q}$ and $H^{r}=L^{r}$. By definition and the Lebesgue dominated convergence theorem, we have

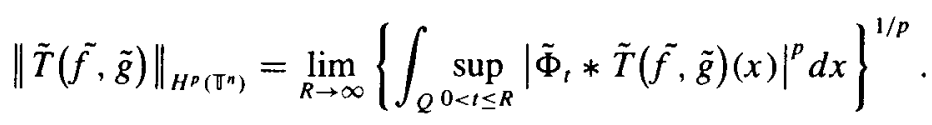

Thus it suffices to show

$$
\left\{\int \sup _{Q<<\leq R}\left|\tilde{\Phi}_{t} * \tilde{T}(\tilde{f}, \tilde{g})(x)\right|^{p} d x\right\}^{1 / p} \leq C\|\tilde{f}\|_{L^{q}\left(\mathbb{J}^{n}\right)}\|\tilde{g}\|_{L^{r}\left(\mathbb{J}^{n}\right)}
$$

with $C$ being independent of $R>0$.

By definition, it is easy to check that, for each fixed $t>0$,

$$
\begin{aligned}
& \tilde{\Phi}_{t} * \tilde{T}(\tilde{f}, \tilde{g})(x)=\sum_{k} \sum_{\nu} a_{k} b_{v} \lambda(k, v) \hat{\Phi}(t(k+v)) e^{2 \pi i\langle k+v, x\rangle}, \\
& \Phi_{t} * T(f, g)(x)=\iint_{\mathbb{R}^{n} \times \mathbb{R}^{n}} \hat{f}(u) \hat{g}(v) \lambda(u, v) \hat{\Phi}(t(u+v)) e^{2 \pi i(u+v, x\rangle} d u d v .
\end{aligned}
$$


Let $\Omega_{K}$ and $\Psi^{1 / N}(x)$ be the same as in Section 3. For each $t>0$, using $\tilde{\Phi}_{t} * \tilde{T}$ and $\Phi_{t} * T$ instead of $\tilde{T}$ and $T$ in (3.1), respectively, we obtain

$$
\begin{aligned}
E_{N, r}(\tilde{f}, \tilde{g})(x)= & \Psi(x / N)^{2} \tilde{\Phi}_{\imath} * \tilde{T}(\tilde{f}, \tilde{g})(x)-\Phi_{\imath} * T\left(\Psi^{1 / N} \tilde{f}, \Psi^{1 / N} \tilde{g}\right)(x) \\
= & -\sum_{k \in \Lambda} \sum_{\nu \in \Lambda} a_{k} b_{\nu} e^{2 \pi i(k+\nu, x)} \int_{\mathbb{R}^{n}} \int_{\mathbb{R}^{n}} \hat{\Psi}(u) \hat{\Psi}(v) \\
& \times\{\lambda(k+u / N, \nu+v / N) \hat{\Phi}(t(k+u / N+v+v / N)) \\
& \quad-\hat{\Phi}(t(k+v)) \lambda(k, v)\} e^{2 \pi i(u, x / N)} e^{2 \pi i(v, x / N\rangle} d u d v .
\end{aligned}
$$

Since $\left\{a_{k}\right\},\left\{b_{\nu}\right\}$ decay rapidly, $\lambda$ and $\hat{\Phi}$ are $L^{\infty}$ and continuous, it is clear that $E_{N, t}(\tilde{f}, \tilde{g})(x) \rightarrow 0$ uniformly for $x \in \mathbb{R}^{n}$ and $t \in[0, R]$ as $N \rightarrow \infty$. Thus we can obtain (4.5) by emulating the proof of Theorem 3.

CASE 2. $0<p \leq 1$ and $0<q, r \leq 1$.

We note that, for a $C^{\infty}$ function $\tilde{f(x)}=\sum a_{k}(\tilde{f}) e^{2 \pi i\langle k, x)}$,

and

$$
\left\|a_{0}(\tilde{f})\right\|_{H^{p}\left(\mathbf{T}^{n}\right)}=C\left|a_{0}(\tilde{f})\right|=\left|\int_{Q} \tilde{f}(x) d x\right|
$$

Thus

$$
\sup _{t>0}\left|\tilde{\Phi}_{t} * \tilde{f}(x)\right| \geq \lim _{t \rightarrow \infty}\left|\tilde{\Phi}_{t} * \tilde{f}(x)\right|=\left|a_{0}(\tilde{f})\right| \text {. }
$$

$$
\left\|a_{0}(\tilde{f})\right\|_{H^{p}\left(\mathbf{T}^{n}\right)}^{p}=\int_{Q}\left\|a_{0}(\tilde{f})\right\|_{H^{p}\left(\mathbb{J}^{n}\right)}^{p} d x \leq C \int_{Q} \sup _{t>0}\left|\tilde{\Phi}_{t} * \tilde{f}(x)\right|^{p} d x=C\|\tilde{f}\|_{H^{p}\left(\mathbb{J}^{n}\right)}^{p} .
$$

Because we can write

and

$$
\tilde{f(x)}=a_{0}+\sum_{k \neq 0} a_{k} e^{2 \pi i(k, x)}=a_{0}+\tilde{f_{1}}(x)
$$

$$
\tilde{g}(x)=b_{0}+\sum_{\nu \neq 0} b_{\nu} e^{2 \pi i\langle v, x\rangle}=b_{0}+\tilde{g}_{1}(x),
$$

we treat $\tilde{T}\left(a_{0}, b_{0}\right), \tilde{T}\left(a_{0}, \tilde{g}_{1}\right), \tilde{T}\left(\tilde{f_{1}}, b_{0}\right)$ and $\tilde{T}\left(\tilde{f_{1}}, \tilde{g}_{1}\right)$ separately. The first one is easily estimated by the above observation. To estimate the last one, we write $\tilde{f}$ and $\tilde{g}$ for $\tilde{f}_{1}$ and $\tilde{g}_{1}$, respectively, for the sake of simplicity. Then we have $\int_{Q} \tilde{f} d x=\int_{Q} \tilde{g} d x=0$ so that by Lemma 1 , we can write $\tilde{f}$ and $\tilde{g}$ in the forms of their atomic decompositions

$$
\tilde{f}(x)=\sum c_{k} \alpha_{k}(x), \quad \tilde{g}(x)=\sum \beta_{\nu} O_{\nu}(x),
$$

where each $\alpha_{k}$ is a $(q, 2,[n(1 / q-1)]+2 n)$ atom and each $O_{v}$ is a $(r, 2,[n(1 / r-$ $1)]+2 n)$ atom, and

$$
\sum\left|c_{k}\right|^{q} \cong\|\tilde{f}\|_{H^{q}\left(\mathbb{J}^{n}\right)}^{q}, \quad \sum\left|\beta_{\nu}\right|^{r} \cong\|\tilde{g}\|_{H^{r}\left(\mathbb{T}^{n}\right)}^{r} .
$$


We take

$$
\Psi(x)=\prod_{j=1}^{n}\left(1-4 x_{j}^{2}\right)_{+}, \quad \text { where } \quad f_{+}(x)= \begin{cases}f(x) & \text { if } f(x) \geq 0, \\ 0 & \text { if } f(x)<0 .\end{cases}
$$

For positive integers $M$ and $N$, we denote the cube $[-N / 2 M, N / 2 M)^{n}$ by $N Q / M$. For large $N$, by (4.6) and the assumption of the theorem, we have

$$
\begin{aligned}
& \left\|\sup _{0<t \leq R}\left|\tilde{\Phi}_{t} * \tilde{T}(\tilde{f}, \tilde{g})\right|\right\|_{L^{p\left(\mathbb{J}^{n}\right)}} \\
& \quad \cong\left\{N^{-n} \int_{N Q / 2} \Psi^{2}(x / N) \sup _{0<t \leq R}\left|\tilde{\Phi}_{t} * \tilde{T}(\tilde{f}, \tilde{g})(x)\right|^{p} d x\right\}^{1 / p} \\
& \quad \leq C\left\{N^{-n} \int_{R^{n}} \sup _{0<t \leq R}\left|\Phi_{t} * T\left(\tilde{f} \Psi^{1 / N}, \tilde{g} \Psi^{1 / N}\right)(x)\right|^{p} d x\right\}^{1 / p}+o(1) .
\end{aligned}
$$

This shows that

$$
\begin{aligned}
& \left\|\sup _{0<t \leq R} \mid \tilde{\Phi}_{t} * \tilde{T}(\tilde{f}, \tilde{g})\right\| \|_{L^{p}\left(\mathbb{J}^{n}\right)} \\
& \quad \leq C N^{-n / p}\left\|\tilde{f} \Psi^{1 / N}\right\|_{H^{q}\left(\mathbb{R}^{n}\right)}\left\|\tilde{g} \Psi^{1 / N}\right\|_{H^{r}\left(\mathbb{R}^{n}\right)}+o(1), \quad \text { as } N \rightarrow \infty .
\end{aligned}
$$

Therefore, it suffices to show that

$$
\liminf _{N \rightarrow \infty} N^{-n / p}\left\|\tilde{f} \Psi^{1 / N}\right\|_{H^{q}\left(\mathbb{R}^{n}\right)}\left\|\tilde{g} \Psi^{1 / N}\right\|_{H^{r}\left(\mathbb{R}^{n}\right)} \leq C\|\tilde{f}\|_{H^{a}\left(\mathbb{I}^{n}\right)}\|\tilde{g}\|_{H^{r}\left(\mathbb{J}^{n}\right)} .
$$

We note that

$$
\begin{gathered}
\left\|\tilde{f} \Psi^{1 / N}\right\|_{H^{q}\left(\mathbb{R}^{n}\right)}^{q} \leq C \sum_{k}\left|c_{k}\right|^{q}\left\|\alpha_{k} \Psi^{1 / N}\right\|_{H^{q}\left(\mathbb{R}^{n}\right)}^{q}, \\
\left\|\tilde{g} \Psi^{1 / N}\right\|_{H^{r}\left(\mathbb{R}^{n}\right)}^{r} \leq C \sum_{\nu}\left|\beta_{\nu}\right|^{r}\left\|O_{\nu} \Psi^{1 / N}\right\|_{H^{r}\left(\mathbb{R}^{n}\right)}^{r} .
\end{gathered}
$$

Thus we only need to prove that for any $(q, 2, s)$ periodic atom $\alpha(x)$ with support in $B\left(x_{0}, \rho\right) \subset Q$,

$$
\left\|\alpha \Psi^{1 / N}\right\|_{H^{q}\left(\mathbb{R}^{n}\right)} \leq C N^{n / q},
$$

where $C$ is a constant independent of $\alpha(x)$ and $N$. By the definition, we have

$$
\begin{aligned}
\left\|\alpha \Psi^{1 / N}\right\|_{H^{q}\left(\mathbb{R}^{n}\right)}^{q} & \cong \int_{\mathbb{R}^{n}} \sup _{0<t<\infty}\left|\int_{\mathbb{R}^{n}} \Psi(x / N) \alpha(x) \Phi_{t}(y-x) d x\right|^{q} d y \\
& =\int_{\mathbb{R}^{n}} \sup _{0<t<\infty}\left|\int_{\mathbb{R}^{n}} \prod_{j=1}^{n}\left(1-4 x_{j}^{2} / N^{2}\right)_{+} \alpha(x) \Phi_{t}(y-x) d x\right|^{q} d y \\
& =\int_{\mathbb{R}^{n}} \sup _{0<t<\infty}\left|\int_{\left|x_{j}\right|<N / 2}\left\{\prod_{j=1}^{n}\left(1-4 x_{j}^{2} / N^{2}\right) \alpha(x)\right\} \Phi_{t}(y-x) d x\right|^{q} d y .
\end{aligned}
$$


Now if we let $N=2 m+1$, then, up to a set of measure 0 , the set $\left\{x \in \mathbb{R}^{n}\right.$ : $\left.\left|x_{j}\right|<m+1 / 2, j=1,2, \ldots, n\right\}$ is the union of the disjoint sets $\{Q+k: k=$ $\left.\left(k_{1}, \ldots, k_{n}\right),-m \leq k_{j} \leq m, j=1,2, \ldots, n\right\}=\left\{Q_{k}\right\}$, where the $k_{j}$ 's are integers. Now the last integral above is bounded by

$$
I_{m}=C \sum_{-m \leq k_{j} \leq m} \int_{\mathbb{R}^{n}} \sup _{0<t<\infty}\left|\int_{Q_{k}}\left\{\prod_{j=1}^{n}\left(1-4 x_{j}^{2} / N^{2}\right) \alpha(x)\right\} \Phi_{t}(y-x) d x\right|^{q} d y .
$$

Noting that $\alpha(x)$ is a periodic function, we easily see that $\chi_{Q_{k}}(x) \alpha(x)$ is an atom with support in $Q_{k}$. Also since on $Q_{k}, \prod_{j=1}^{n}\left(1-4 x_{j}^{2} / N^{2}\right)$ is a polynomial of degree $2 n$ which is bounded by 1 , clearly

$$
A(x)=\prod_{j=1}^{n}\left(1-4 x_{j}^{2} / N^{2}\right) \chi_{Q_{k}}(x) \alpha(x)
$$

is a $(q, 2,[n(1 / q-1)])$ atom on $\mathbb{R}^{n}$. So by a well-known estimate, the above integral $I_{m}$ is bounded by

$$
C \sum_{-m \leq k_{j} \leq m}\|A\|_{H^{q}\left(\mathbb{R}^{n}\right)}^{q} \leq C N^{n},
$$

which shows $\left\|\alpha \Psi^{1 / N}\right\|_{H^{q}\left(\mathbb{R}^{n}\right)} \leq C N^{n / q}$.

Finally we treat $\tilde{T}\left(\tilde{f_{1}}, b_{0}\right)$. Let $\Gamma$ be a $C^{\infty}$ function supported in $\Omega_{K}=[-1 / 2-$ $1 / K, 1 / 2+1 / k]$ for some fixed positive constant $K$. Suppose that $\Gamma(x) \equiv 1$ on $Q$ and $\left\|\Gamma^{1 / N}\right\|_{H^{\prime}\left(\mathbb{R}^{n}\right)} \leq C N^{n / r}$. (We assume a suitable cancellation condition to get the last property of $\Gamma$.) Let $\Psi$ be as above. Put

$$
\begin{aligned}
E_{N . t}(\tilde{f}, \tilde{g})(x)= & \Psi(x / N) \Gamma(x / N) \tilde{\Phi}_{t} * \tilde{T}(\tilde{f}, \tilde{g})(x)-\tilde{\Phi}_{t} * T\left(\Psi^{1 / N} \tilde{f}, \Psi^{1 / N} \tilde{g}\right)(x) \\
= & -\sum_{k \in \Lambda} \sum_{v \in \Lambda} a_{k} b_{v} e^{2 \pi i(k+v, x)} \int_{\mathbb{R}^{n}} \int_{\mathbb{R}^{n}} \hat{\Psi}(u) \hat{\Gamma}(v) \\
& \times\{\lambda(k+u / N, v+v / N) \hat{\Phi}(t(k+u / N+v+v / N)) \\
& \quad-\lambda(u, v) \hat{\Phi}(t(k+v))\} e^{2 \pi i\langle u, x / N\rangle} e^{2 \pi i\langle v, x / N\rangle} d u d v .
\end{aligned}
$$

Then, for any fixed $R>0, E_{N, t}(\tilde{f}, \tilde{g})(x) \rightarrow 0$ uniformly in $x \in \mathbb{R}^{n}$ and $t \in[0, R]$ as $N \rightarrow \infty$. Therefore, arguing as in (4.7), we have

$$
\begin{aligned}
& \left\|\sup _{0<t \leq R}\left|\tilde{\Phi}_{t} * \tilde{T}\left(\tilde{f_{1}}, b_{0}\right)\right|\right\|_{L^{p\left(\mathbb{T}^{n}\right)}}\|\| b_{0} \Gamma^{1 / N} \|_{H^{r}\left(\mathbb{R}^{n}\right)}+o(1), \quad \text { as } N \rightarrow \infty . \\
& \quad \leq C N^{-n / p}\left\|\tilde{f} \Psi^{1 / N}\right\|_{H^{q}\left(\mathbb{R}^{n}\right)}
\end{aligned}
$$

By (4.10) and the estimates on (4.8), we see that the left-hand side is bounded by $C\|\tilde{f}\|_{H^{q}\left(\mathbb{T}^{n}\right)}\|\tilde{g}\|_{H^{r}\left(\mathbb{T}^{n}\right)}$. Clearly, we have the same estimate for $\tilde{T}\left(a_{0}, \tilde{g}_{1}\right)$. 
CASE 3. $0<p \leq 1,0<q \leq 1<r$. The proof for this case is an easy combination of those for Cases 1 and 2, we leave the proof to the reader.

The following theorem is the converse of Theorem 5 .

THEOREM 6. Let $\lambda$ and $p, q, r$ be as in Theorem 5 , and $T(f, g)$ and $\tilde{T}_{\varepsilon}(\tilde{f}, \tilde{g})$ be as in Section 3. If there is $a C>0$ such that

$$
\left\|\tilde{T}_{\varepsilon}(\tilde{f}, \tilde{g})\right\|_{H^{p}\left(\mathbb{T}^{n}\right)} \leq C\|\tilde{f}\|_{H^{a}\left(\mathbb{T}^{n}\right)}\|\tilde{g}\|_{H^{r}\left(\mathbb{J}^{n}\right)} \text { for all } f, g \in C^{\infty}\left(\mathbb{T}^{n}\right)
$$

uniformly for $\varepsilon>0$, then

$$
\|T(f, g)\|_{H^{p}\left(\mathbb{R}^{n}\right)} \leq C\|f\|_{H^{q}\left(\mathbb{R}^{n}\right)}\|g\|_{H^{r}\left(\mathbb{R}^{n}\right)}
$$

for all $f \in H^{q}\left(\mathbb{R}^{n}\right) \cap \mathscr{S}\left(\mathbb{R}^{n}\right)$ and $g \in H^{r}\left(\mathbb{R}^{n}\right) \cap \mathscr{S}\left(\mathbb{R}^{n}\right)$.

To prove Theorem 6 , we need the following lemma.

LEMMA 2. Let $f \in H^{p}\left(\mathbb{R}^{n}\right) \cap \mathscr{S}\left(\mathbb{R}^{n}\right)$, and define $\tilde{f_{\varepsilon}}$ as in (2.1). Then

$$
\lim _{\varepsilon \rightarrow 0} \varepsilon^{n(1-1 / p)}\left\|\tilde{f_{\varepsilon}}\right\|_{H^{p}\left(\mathbb{T}^{n}\right)}=\|f\|_{H^{p}\left(\mathbb{R}^{n}\right)} .
$$

See [LL, Lemma 3] for a proof.

Now we return to prove Theorem 6. Let $f \in H^{q}\left(\mathbb{R}^{n}\right) \cap \mathscr{S}\left(\mathbb{R}^{n}\right)$ and $g \in H^{r}\left(\mathbb{R}^{n}\right) \cap$ $\mathscr{S}\left(\mathbb{R}^{n}\right)$. As in the proof of Theorem 1, we have

$$
\lim _{\varepsilon \rightarrow 0} \varepsilon^{2 n} \tilde{\Phi}_{t \varepsilon} * \tilde{T}_{\varepsilon}\left(\tilde{f_{\varepsilon}}, \tilde{g}_{\varepsilon}\right)(\varepsilon x)=\Phi_{t} * T(f, g)(x)
$$

by the definition of the Riemann integral. Let $\eta$ be as in the proof of Theorem 1. By Fatou's lemma we see that

$$
\begin{aligned}
\|T(f, g)\|_{H^{p}\left(\mathbb{R}^{n}\right)}^{p} & =\int_{\mathbb{R}^{n}} \sup _{t>0}\left|\Phi_{t} * T(f, g)(x)\right|^{p} d x \\
& \leq \liminf _{\varepsilon \rightarrow 0} \varepsilon^{2 n p} \int_{\mathbb{R}^{n}} \eta(\varepsilon x) \sup _{t>0}\left|\tilde{\Phi}_{t \varepsilon} * \tilde{T}_{\varepsilon}\left(\tilde{f_{\varepsilon}}, \tilde{g}_{\varepsilon}\right)(\varepsilon x)\right|^{p} d x \\
& \leq \liminf _{\varepsilon \rightarrow 0} \varepsilon^{2 n p-n} \int_{Q} \sup _{t>0}\left|\tilde{\Phi}_{t} * \tilde{T}_{\varepsilon}\left(\tilde{\tilde{f}_{\varepsilon}}, \tilde{g}_{\varepsilon}\right)(x)\right|^{p} d x .
\end{aligned}
$$

By the assumption and Lemma 2, we have

$$
\begin{aligned}
\|T(f, g)\|_{H^{p}\left(\mathbb{R}^{n}\right)}^{p} & \leq C^{p} \liminf _{\varepsilon \rightarrow 0} \varepsilon^{2 n p-n}\left\|\tilde{f_{\varepsilon}}\right\|_{H^{a}\left(\mathbb{I}^{n}\right)}^{p}\left\|\tilde{g}_{\varepsilon}\right\|_{H^{r}\left(\mathbb{J}^{n}\right)}^{p} \\
& \leq C^{p}\|\tilde{f}\|_{H^{q}\left(\mathbb{R}^{n}\right)}^{p}\|\tilde{g}\|_{H^{r}\left(\mathbb{R}^{n}\right)^{p}}^{p}
\end{aligned}
$$


This completes the proof of Theorem 6.

Finally, we point out an application of Theorem 5. Suppose that $B_{1}(f, g)$ and $\tilde{B}_{1}(\tilde{f}, \tilde{g})$ are defined as in (4.1) and (4.2), with $U_{1}$ and $V_{1}$ being standard CalderónZygmund operators. Also assume

$$
\left(\widehat{\left.U_{1} f\right)(u)}=\hat{f}(u) \lambda_{1}(u), \quad\left(\widehat{\left.V_{1} g\right)(v)}=\hat{g}(v) \mu_{1}(v),\right.\right.
$$

where both $\lambda_{1}$ and $\mu_{1}$ are continuous and bounded. By results in [CG] and [G] and Theorem 5, we have

THEOREM 7. Let $0<q, r \leq 1$ and $1 / p=1 / r+1 / q$. Assume that for some non-negative integer $\kappa$, there is an such that

$$
\int_{\mathbb{R}^{n}} x^{\beta} B_{1}(f, g) d x=0
$$

for all multi-indices $\beta$ with $|\beta| \leq \kappa$, and all $(r, 2, s)$ atoms $g$ and $(q, 2, s)$ atoms $f$. Then for $n /(n+\kappa+1)<p \leq 1, \tilde{B}_{1}(\tilde{f}, \tilde{g})$ can extend to a bounded operator from $H^{q}\left(\mathbb{J}^{n}\right) \times H^{r}\left(\mathbb{T}^{n}\right)$ into $H^{p}\left(\mathbb{T}^{n}\right)$.

\section{References}

[AC] P. Auscher and M. J. Carro, 'On relations between operators on $\mathbb{R}^{n}, \mathbb{T}^{n}$ and $\mathbb{Z}^{n}$, Studia Math. 101 (1992), 165-182.

[BF] B. Blank and D. Fan, ' $S$-functions and $g_{\lambda}$-functions on compact Lie groups', J. Austral. Math. Soc. (Series A) 61 (1996), 327-344.

[CG] R. Coifman and L. Grafakos, 'Hardy space estimates for multilinear operators (I)', Rev. Mat. Iberoamericana 8 (1992), 45-62.

[CM1] R. Coifman and Y. Meyer, 'On commutators of singular integrals and bilinear singular integrals', Trans. Amer. Math. Soc. 212 (1975), 315-331.

[CM2] - 'Commutateurs d'integrales singulières et opérateurs multilinéaires', Ann. of Inst. Fourier (Grenoble) 28 (1978), 177-202.

[CM3] - Au-delà des opérateurs pseudo-différentiels, Astérisque 57 (Société Mathématique de France, Paris, 1978).

[CM4] - 'Non-linear harmonic analysis, operator theory and P.D.E.', Beijing Lectures in Analysis, Ann. of Math. Stud. 112 (Princeton Univ. Press, Princeton, 1986), pp. 3-46.

[F] D. Fan, 'Multipliers on certain function spaces', Rend. Circ. Mat. Palermo (2) 43 (1994), $449-463$.

[FS] G. Folland and E. M. Stein, Hardy spaces on homogeneous groups (Princeton Univ. Press, Princeton, 1982).

[G] L. Grafakos, 'Hardy space estimates for multilinear operators (II)', Rev. Mat. Iberoamericana 8 (1992), 69-92.

[GK] L. Grafakos and N. Kalton, 'Some remarks on multilinear maps and interpolation', to appear. 
[GT] L. Grafakos and R. Torres, 'Multilinear Calderón-Zygmund theory', to appear.

[GW] L. Grafakos and G. Weiss, 'Transference of multilinear operators', Illinois J. Math. 40 (1996), 344-351.

[K] M. Kaneko, 'Boundedness of some operators composed by Fourier multipliers', Tohoku Math. J. 35 (1983), 267-288.

[KaS] M. Kaneko and E. Sato, 'Notes on transference of continuity from maximal Fourier multiplier operators on $\mathbb{R}^{n}$ to those on $\mathbb{T}^{n}$, Interdiscip. Inform. Sci. 4 (1998), 97-107.

[KeS] C. Kenig and E. M. Stein, 'Multilinear estimates and fractional integration', Math. Research Lett. 6 (1999), 1-15.

[KT] C. Kenig and P. Thomas, 'Maximal operators defined by Fourier multiplier', Studia Math. 68 (1980), 79-83.

[L] K. DeLeeuw, 'On $L^{p}$ multiplier', Ann. of Math. 91 (1965), 364-379.

[La] M. Lacey, 'On the bilinear Hilbert transform', in: Proceedings of International Congress of Mathematicians, vol. II, (Berlin, 1998), Doc. Math. 1998, Extra vol. II, pp. 647-656.

[LT] M. Lacey and C. Thiele, ' $L^{p}$ estimates on the bilinear Hilbert transform', Ann. of Math. (2) 146 (1997), 693-724.

[LL] Z. Liu and S. Lu, 'Transference and restriction of maximal multiplier operators on Hardy spaces', Studia Math. 105 (1993), 121-134.

[S] E. M. Stein, Harmonic analysis, real-variable methods, orthogonality, and oscillatory integrals (Princeton Univ. Press, Princeton, 1993).

[SW] E. M. Stein and G. Weiss, Introduction to Fourier analysis on Euclidean spaces (Princeton Univ. Press, Princeton, 1971).

[T] R. Torres, 'Spaces of sequence, sampling theorems and functions of exponential type', Studia Math. 100 (1991), 51-74.

Department of Mathematics

Huazhong University of

Science and Technology

and

Department of Mathematics

University of Wisconsin-Milwaukee

Milwaukee, WI 53201

USA

e-mail: fan@csd.uwm.edu
Department of Mathematics

Kanazawa University

Kanazawa 920-11

Japan

e-mail: shuichi@kenroku.kanazawa-u.ac.jp 